\title{
Der Nachweis toxischer Basen im Harn.
}

IV. Mitteilung.

Von

Fr. Kutscher.

(Aus dem Physiologischen Institut der Universität Marburg.)

(Der Redaktion zugegangen am 3. April 1907.)

In einigen Abhandlungen $\left.{ }^{1}\right)$ konnten Lohmann und Kutscher über eine Anzahl bisher nicht darstellbarer Harnbasen berichten. Am besten hatten sich uns zur Trennung der verschiedenen Basen von einander die Goldsalze bewährt. Nach Entfernung der leichter krystallisierbaren Goldverbindungen hinterblieb jedoch ein nicht unbeträchtlicher Rest, der sich immer wieder ölig abschied. Über die Gewinnung des goldhaltigen Öles aus $100 \mathrm{l}$ Frauenharn gibt unsere Mitteilung III genaue Auskunft. Es ist mir nun gelungen, das Gemenge der Basen, das in dem Öl steckte, fast restlos aufzuteilen und in gut krystallisierende Körper überzuführen. Die befolgte Methode war folgende.

Die ölig abgeschiedenen Goldverbindungen wurden in heißem Wasser unter Zugabe einiger Tropfen starker Salzsäure gelöst und durch Schwefelwasserstoff von Gold befreit, die freigewordenen Chloride zum dünnen Sirup eingeengt. Sie wurden dann einige Tage im Exsikkator über Schwefelsäure gehalten, hier erstarrten sie bald zu einem Krystallbrei. Derselbe wurde mit kaltem absoluten Alkohol verrieben, dabei blieb der kleinere Teil ungelöst. Der Rückstand bestand aus langen weißen Nadeln. Dieselben wurden abgesaugt, mit Alkohol gewaschen, in wenig Wasser gelöst und mit 30\% \%iger wässeriger Goldchloridlösung gefällt. Das ausgefallene Goldsalz wurde aus wenig heißem, salzsäurehaltigem Wasser umkrystallisiert. Es schied sich beim

1) Diese Zeitschrift, Bd. XLVIII, S. 1 u. 422 ; Bd. XLIX, S. 81.

Hoppe-Seyler's Zeitschrift f. physiol. Chemie. LI. 
Erkalten der Flüssigkeit sofort in kleinen, durchsichtigen, gelbroten, vierseitigen Säulen ab.

Die Ausbeute an Goldsalz betrug ca. $0,45 \mathrm{~g}$. Die Goldverbindung schmolz scharf bei $194^{\circ}$ C. zu einer klaren rotbraunen Flüssigkeit. Das Chlorid schmolz bis $300^{\circ}$ erhitzt nicht. Es färbte sich nur schwarz.

Die Analyse des Goldsalzes gab folgende Werte:

$$
\begin{aligned}
& 0,1279 \mathrm{~g} \text { gaben } 0,0551 \mathrm{~g} \mathrm{Au} \text {. } \\
& 0,1279 " 》 \quad 0,0242 \mathrm{~g} \mathrm{H}_{2} \mathrm{O} \text { und } 0,0816 \mathrm{~g} \mathrm{CO}_{2} . \\
& 0,1203 》 " 4,2 \mathrm{~cm} \mathrm{~N} ; \mathrm{T} .=11^{\circ} ; \mathrm{Ba} .=741 \mathrm{~mm} .
\end{aligned}
$$

Die gefundenen Zahlen stimmen am besten zu der Formel:

$$
\mathrm{C}_{13} \mathrm{H}_{18} \mathrm{~N}_{2} \mathrm{O}_{2} \cdot 2 \mathrm{HCl} \cdot 2 \mathrm{AuCl}_{3} \text {. }
$$

Leider ermöglichte die geringe Menge der mir zur Verfügung stehenden Substanz es nicht, durch Umkrystallisation mit nachfolgender Goldbestimmung ihre Reinheit völlig sicher zu stellen. Ich nenne die Base, deren Chlorid und Goldsalz sich hatte darstellen lassen, «Mingin».

Berechnet:

Für $\mathrm{C}_{18} \mathrm{H}_{18} \mathrm{~N}_{2} \mathrm{O}_{2} \cdot 2 \mathrm{HCl} \cdot 2 \mathrm{AuCl}_{3}$

$$
\begin{array}{ll}
\mathrm{C}=17,1 \% & \mathrm{C}=17,4 \% \\
\mathrm{H}=2,2 \% & \mathrm{H}=2,1 \% \\
\mathrm{~N}=3,1 \% & \mathrm{~N}=4,0 \% \\
\mathrm{Au}=43,1 \% & \mathrm{Au}=43,1 \%
\end{array}
$$

Gefunden:

Das alkoholische Filtrat vom Minginchlorid wurde mit alkoholischer Quecksilberchloridlösung gefällt und durch Eintragen von fein gepulvertem Quecksilberchlorid Sorge getragen, daß die Flüssigkeit sich mit Sublimat sättigte. Der gesamte Niederschlag wurde nach 48 Stunden abgesaugt, mit alkoholischer Quecksilberchloridlösung gewaschen, in heißem Wasser gelöst, mit Schwefelwasserstoff zersetzt. Die so erhaltenen Chloride wurden stark eingeengt und mit 30\% iger wässeriger Goldchloridlösung gefällt. Es schied sich zunächst ein Öl ab, das nach einigen Tagen krystallisierte. Nach zweimaliger Umkrystallisation war die Goldverbindung analysenrein. Aber auch in reinem Zustande scheidet sie sich aus ihren Lösungen immer erst in Form öliger Tropfen ab, die sich langsam (in 24-48 Stunden), jedoch vollkommen in kleine Drusen von Blättchen und kurzen 
Nadeln umwandeln. Wie die Analyse und die weitere Untersuchung ergab, lag in der isolierten Verbindung ein Körper vor, der zum Novain anscheinend in demselben Verhältnisse steht, wie das Neurin zum Cholin. Ich bezeichne deshalb diese neue von mir aufgefundene Verbindung als "Reduktonovain . Die Quecksilberfällung bestand fast ausschließlich aus Reduktenovain.

Die Goldverbindung des Reduktonovains ist in kaltem Wasser schwer löslich. Will man sie umkrystallisieren, so muß man stark salzsäurehaltiges Wasser benutzen, da man anders reichliche Ausscheidung von metallischem Gold bekommt. In heißem, salzsäurehaltigem Wasser ist sie ziemlich leicht löslich. Sie scheidet sich daraus, wie bereits gesagt, zunächst als Öl ab. Gegen Licht ist sie empfindlich. Schon bei $80^{\circ}$ beginnt sie etwas zu sintern und sich zu verfärben, ohne jedoch an Gewicht zu verlieren. Selbst Substanz, die ich zur Analyse bei $110^{\circ}$ getrocknet hatte und die sehr dunkel geworden war, zeigte keinen Gewichtsverlust. Im Schmelzröhrchen kann man stärkeres Sintern gegen $125^{\circ}$ beobachten, zwischen $155-160^{\circ}$ schmilzt sie, wird aber erst zwischen $175-180^{\circ}$ ganz klar. Über $180^{\circ}$ erhitzt beginnt sie Blasen zu werfen. Die Ausbeute an analysenreinem Reduktonovaingoldchlorid hatte ca. 2,0 $\mathrm{g}$ betragen. Die Analyse gab folgende Werte:

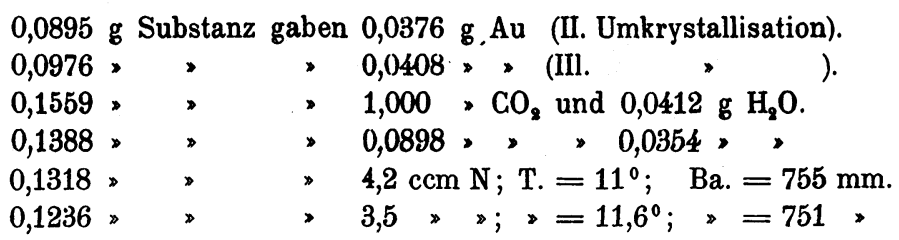

Für $\mathrm{C}_{7} \mathrm{H}_{16} \mathrm{NOCl} \cdot \mathrm{AuCl}_{8}$

Berechnet:

$$
\begin{aligned}
& \mathrm{C}=17,9 \% \\
& \mathrm{H}=3,4 \% \\
& \mathrm{~N}=3,0 \% \\
& \mathrm{Au}=42,1 \%
\end{aligned}
$$

Gefunden :

$$
\begin{aligned}
& \mathrm{C}=17,5 ; 17,6 \% \\
& \mathrm{H}=3,0 ; 2,9 \% \\
& \mathrm{~N}=3,7 ; 3,4 \% \\
& \mathrm{Au}=42,0 ; 41,8 \%
\end{aligned}
$$

War meine Voraussetzung über die nahen Beziehungen der neuen Base zum Novain, die ich durch ihren Namen angedeutet habe, richtig, dann ließ sich erwarten, daß auch das 
Reduktonovain beim Behandeln mit Alkali Trimethylamin abspalten würde. Das ist nun in der Tat der Fall. Bei der Spaltung des Reduktonovains verfuhr ich wie bei der Zersetzung des Novains.

$0,618 \mathrm{~g}$ analysenreines Reduktonovaingoldchlorid wurden mit Schwefelwasserstoff zersetzt, das in Freiheit gesetzte Chlorid auf dem Wasserbade eingeengt. Es krystallisierte bereits auf dem Wasserbade in langen, glänzenden Nadeln. Der krystallinische Rückstand wurde mit $20 \mathrm{~g}$ festem Barythydrat destilliert, das Destillat in konzentrierter Salzsäure aufgefangen. Nach dem Verdunsten des Destillates verblieben hygroskopische Krystalle, aus denen sich unschwer mit Hilfe von Goldchlorid die gut krystallisierende, schwer lösliche Goldverbindung des Trimethylamins darstellen ließ. Dieselbe lieferte bei der Analyse folgenden Wert.

0,1048 g Substanz gaben $0,0517 \mathrm{~g} \mathrm{Au}$.

Für $\mathrm{N} \cdot\left(\mathrm{CH}_{3}\right)_{8} \cdot \mathrm{HCl} \cdot \mathrm{AuCl}_{3}$

Berechnet:

$\mathrm{Au}=49,4 \%$
Gefunden:

$\mathrm{Au}=49,3 \%$

Durch diesen Versuch ist die Zugehörigkeit des Reduktonovains $\mathrm{zu}$ den Cholinbasen erwiesen und es ist dem Neosin, Novain und Oblitin anzureihen. Von den genannten Basen hat sich das Neosin bisher im Harn nicht auffinden lassen. Dagegen konnte gezeigt werden, daß das Novain und Oblitin den Tierkörper passieren und im Harne erscheinen können. Namentlich ließ sich nach Verfütterung des von einer englischen Aktiengesellschaft vertriebenen «Extractum carnis Liebig» an Hunde im Harn der gefütterten Tiere Novain ${ }^{1}$ ) nachweisen. Auch aus menschlichem Harn ist das Novain bereits, sogar vor dem genaueren Bekanntwerden dieser Base, durch Stéphane Da-

1) Das aus Hundeharn dargestellte Novaingoldchlorid schmolz bei $132-135^{\circ}$. Ich hielt es deshalb nïcht für ganz rein. In der Tat war es ganz rein. Inzwischen habe ich Präparate von gleichen Eigenschaften und dem verlangten Goldwert $(40,5 \%)$ auch aus Fleischextrakt darstellen und die Ursache aufdecken können, warum bej meinen früheren Präparaten aus Fleischextrakt ḋer Goldwert hinter dem verlangten merklich zurückblieb. Ich werde darauf seiner Zeit näher eingehen. 
browski ${ }^{1}$ ) gewonnen worden. Dabrowski isolierte aus $100 \mathrm{l}$ normalen Menschenharn das Platinat einer Base, die er nicht benannt hat. Das Platinat besitzt die Eigenschaften des Novainplatinates. Ihm wird von seinem Entdecker die Formel $\left(\mathrm{C}_{7} \mathrm{H}_{15} \mathrm{NO}_{2} \cdot \mathrm{HCl}\right)_{2} \cdot \mathrm{PtCl}_{4}$ zugeschrieben, während ich für das Novainplatinat die Formel $\left(\mathrm{C}_{7} \mathrm{H}_{18} \mathrm{NO}_{2} \mathrm{Cl}\right)_{2} \cdot \mathrm{PtCl}_{4}$ annehme. Beide Formeln unterscheiden sich also nur im Wasserstoff und es ist nicht ganz leicht, durch die Analyse zu entscheiden, welches die richtige ist. Um das zu erweisen, lasse ich die Prozentberechnung für die beiden Formeln folgen.

Für $\left(\mathrm{C}_{7} \mathrm{H}_{15} \mathrm{NO}_{2} \cdot \mathrm{HCl}\right)_{2} \cdot \mathrm{PtCl}_{4}$ :

$$
\begin{aligned}
& \mathrm{C}=24,0 \% \\
& \mathrm{H}=4,6 \% \\
& \mathrm{~N}=4,0 \% \\
& \mathrm{O}=9,2 \% \\
& \mathrm{Pt}=27,8 \% \\
& \mathrm{Cl}=30,4 \%
\end{aligned}
$$

Für $\left(\mathrm{C}_{7} \mathrm{H}_{18} \mathrm{NO}_{2} \mathrm{Cl}\right)_{2} \cdot \mathrm{PtCl}_{4}$ :

$$
\begin{aligned}
& \mathrm{C}=23,9 \% \\
& \mathrm{H}=5,1 \% \\
& \mathrm{~N}=4,0 \% \\
& \mathrm{O}=9,1 \% \\
& \mathrm{Pt}=27,7 \% \\
& \mathrm{Cl}=30,2 \%
\end{aligned}
$$

Nehmen wir dazu die Fähigkeit des Novains, in den Harn überzutreten, so ist wohl nicht zweifelhaft, daß die unbenannte Base Dabrowskis in der Tat Novain gewesen ist. Jedoch scheint der Mensch in der Regel nicht Novain, sondern Reduktonovain auszuscheiden, denn bereits bei unserer ersten Untersuchung von menschlichem Harn erhielten Lohmann und ich das Goldsalz einer Base, das sich in seinen Analysenzahlen mehr dem Reduktonovaingoldchlorid näherte. Bei meiner jetzigen Untersuchung, wo ich genügend Material zu vollkommenen Analysen bekam, vermißte ich das Novain sogar ganz. Es scheint danach der Organismus des Menschen die Fähigkeit zu besitzen, das Novain vollständig in Reduktonovain überzuführen, während der Hund eine derartige Umwandlung am Novain wahrscheinlich nicht vollziehen kann.

Das Auftreten dieser verschiedenen Cholinbasen im Harn gibt uns auch Aufschluß über eine bereits vor langer Zeit am Harn beobachtete auffällige Erscheinung. Dessaignes ${ }^{2}$ ) stellte im Jahre 1856 als erster fest, daß man durch Destillation von

1) Sur la mannite et les ptomaïnes dans l'urine normale de l'homme, 1903, Sonderabzug.

$\left.{ }^{2}\right)$ Liebigs Annalen, Bd. C, S. 218. 
menschlichem Harn mit fixen Alkalien merkliche Mengen von Trimethylamin erhalten könnte. Bereits Dessaignes sprach die Vermutung aus, das Trimethylamin wäre von komplizierteren Körpern abgespalten. Die Angaben Dessaignes sind vielfach bestätigt worden, doch war es bisher nicht möglich, die Muttersubstanzen des Trimethylamins aus dem Harn darzustellen. Die Frage nach denselben scheint mir jetzt eine befriedigende Lösung gefunden zu haben.

Die letzte Base, die sich außer dem Mingin und Reduktonovain noch isolieren ließ, war durch alkoholische Sublimatlösung nicht fällbar. Sie fand sich im Filtrat der abgesaugten Quecksilberverbindungen des Reduktonovains. Um sie zu isolieren, wurde aus dem Filtrat der Alkohol bei mäßiger Temperatur verjagt, der Rückstand in heißem Wasser gelöst und mit Schwefelwasserstoff zersetzt. Die so gewonnenen Chloride wurden stark eingeengt und mit 30\% oiger wässeriger Goldchloridlösung gefällt. Die Fällung war teils körnig, teils ölig, wurde aber bald bis auf einen geringen Rest krystallinisch. Sie wurde mehrfach aus heißem, salzsäurehaltigem W' und schließlich in gelbroten, glänzenden Blättern und Platten gewonnen. Sie schmolz unscharf bei $167^{\circ}$. Die Schmelze wurde erst bei $190^{\circ}$ klar. Die Analyse wie der Schmelzpunkt zeigte, $\mathrm{da} ß$ es sich um die Goldverbindung einer Base handelte, die ich auch aus Liebigs Fleischextrakt isoliert habe. Ich habe darüber an anderer Stelle berichtet. Die neue Base ist von mir «Vitiatin * genannt worden. Sie ist zweisäurig. Ihr kommt die Formel $\mathrm{C}_{5} \mathrm{H}_{14} \mathrm{~N}_{6}$ zu und ich nehme für sie folgende Konstitution an:

$$
\mathrm{NH}_{2} \cdot \mathrm{CNH} \cdot \underset{\mathrm{CH}_{3}}{\mathrm{~N}} \cdot \mathrm{CH}_{2} \cdot \mathrm{CH}_{2} \cdot \mathrm{NH} \cdot \mathrm{CNH} \cdot \mathrm{NH}_{2} \cdot
$$

Ihre nahen Beziehungen zum Methylguanidin, Dimethylguanidin, Kreatin und Kreatinin leuchten ohne weiteres ein und ihr Auftreten im Harn, der alle ebengenannten Körper enthält, ist nicht auffallend. Die Analyse des Goldsalzes gab folgende Werte:

$0,1730 \mathrm{~g}$ Substanz gaben $0,0465 \mathrm{~g} \mathrm{CO}_{2}$ und $0,0362 \mathrm{~g} \mathrm{H}_{2} \mathrm{O}$.

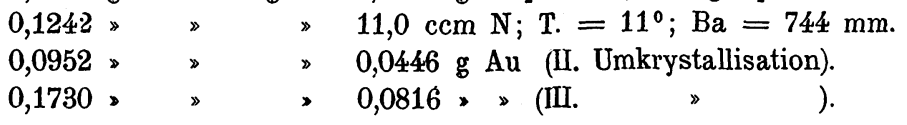




$\begin{array}{cc}\text { Für } & \mathrm{C}_{5} \mathrm{H}_{14} \mathrm{~N}_{\mathbf{6}} \cdot 2 \mathrm{HCl} \cdot 2 \mathrm{AuCl}_{\mathbf{3}} \\ \text { Berechnet: } & \mathrm{Gefunden}: \\ \mathrm{C}=7,2 \% & \mathrm{C}=7,3 \% \\ \mathrm{H}=1,9 \% & \mathrm{H}=2,3 \% \\ \mathrm{~N}=10,0 \% & \mathrm{~N}=10,4 \% \\ \mathrm{Au}=47,0 \% & \mathrm{Au}=46,9 ; 47,2 \%\end{array}$
tragen.

Die Ausbeute an analysenreiner Substanz hatte $0,7 \mathrm{~g}$ be-

Damit haben die Untersuchungen der menschlichen Harnbasen einen gewissen Abschluß erhalten, da sich die durch Phosphorwolframsäure und alkoholische Platinchloridlösung fällbaren annähernd haben aufteilen und festlegen lassen. Ich führe dieselben der Übersicht wegen hier nochmals auf. Es haben sich an bisher unbekannten menschlichen Harnbasen isolieren lassen:

1. Methylguanidin. Das Auftreten von Dimethylguanidin hat sich wahrscheinlich machen lassen. $\mathrm{C}_{2} \mathrm{H}_{7} \mathrm{~N}_{8}$ und $\mathrm{C}_{8} \mathrm{H}_{8} \mathrm{~N}_{8}$.

2. Novain $\mathrm{C}_{7} \mathrm{H}_{18} \mathrm{NO}_{3}$.

3. Reduktonovain $\mathrm{C}_{7} \mathrm{H}_{17} \mathrm{NO}_{2}$.

4. Methylpyridinchlorid $\mathrm{C}_{5} \mathrm{H}_{5} \mathrm{~N} \cdot \mathrm{CH}_{8} \mathrm{Cl}$.

5. Gynesin $\mathrm{C}_{19} \mathrm{H}_{28} \mathrm{~N}_{3} \mathrm{O}_{3}$.

6. Mingin $\mathrm{C}_{18} \mathrm{H}_{18} \mathrm{~N}_{8} \mathrm{O}_{8}$ ?

7. Vitiatin $\mathrm{C}_{5} \mathrm{H}_{14} \mathrm{~N}_{6}$.

Von den neugefundenen Basen hat sich auch mit einiger Sicherheit die Quelle ermitteln lassen. Wir müssen jedenfalls für das Methylguanidin, Dimethylguanidin, Novain, Reduktonovain und Vitiatin einen tierischen Ursprung annehmen, sei es nun, daß sie von den dem Tierreich entstammenden Nahrungs- und Genußmitteln herzuleiten sind, sei es daß sie sich erst von Grund auf im intermediären Stoffwechsel bilden.

Das Methylpyridinchlorid hingegen verdankt pflanzlichen Genußmitteln seine Entstehung, und zwar ist das Pyridin, das der Mensch beim Tabakrauchen und Kaffeetrinken sich zuführt, als Muttersubstanz des Methylpyridins anzusehen. ${ }^{1}$ ) Nur bezüglich des Gynesins und Mingins, für deren Konstitution wir bisher keinen Anhalt haben, befinden wir uns völlig im Ungewissen.

1) Zeitschrift für Nahrungs- und Genußmittel, Bd. XIII, S. 177. 